\title{
Editorial \\ Interferon-induced versus chemokine transcripts as lupus biomarkers
}

\author{
Mary K Crow and Kyriakos A Kirou
}

Mary Kirkland Center for Lupus Research, Hospital for Special Surgery, 535 East 70th Street, New York, NY 10021, USA

Corresponding author: Mary K Crow, crowm@hss.edu

Published: 18 December 2008

Arthritis Research \& Therapy 2008, 10:126 (doi:10.1186/ar2559)

This article is online at http://arthritis-research.com/content/10/6/126

(c) 2008 BioMed Central Ltd

See related research article by Fu et al., http://arthritis-research.com/content/10/5/R112

\begin{abstract}
Compelling support for a central role for interferon-alpha in lupus pathogenesis has led to a new focus on the role of innate immune system activation in the generation of pathogenic mediators. These insights have been extended in translational studies of patients with well-characterized disease activity and clinical manifestations in order to identify informative molecular biomarkers. Chemokines are among the interferon-inducible genes, and new data support an association between the expression of chemokines and both lupus disease activity and organ damage. Longitudinal studies that relate molecular biomarkers to disease activity will be needed to validate these promising data and establish a sensitive measure of change for interventional studies and patient care.
\end{abstract}

The translation of new concepts regarding the innate immune response to the study of patients with systemic lupus erythematosus (SLE) has led to advances in understanding lupus pathogenesis. The identification of biomarkers that reflect those pathogenic mechanisms holds promise of extending those discoveries to improve patient care [1]. Current concepts incorporate a role for endogenous Toll-like receptor ligands, including immune complexes containing host-derived nucleic acids, in the initiation and amplification of immune responses [2-4]. Those immune complexes can induce type I interferon (IFN) and other inflammatory mediators that contribute to autoimmunity and inflammation. Among the functional effects of IFN- $\alpha$ is the generation of proinflammatory mediators, including chemokines that direct the trafficking of inflammatory cells. Through its regulation of chemokines, IFN- $\alpha$ provides a link between innate immune system activation by immune complexes and the characteristic tissue damage seen in SLE patients.

In parallel with progress in defining immunopathogenic mechanisms that underlie SLE, the development of immune systemtargeted therapeutics is increasing activity in interventional clinical trials. Unfortunately, the drug development process in SLE faces daunting challenges. The inherent heterogeneity of lupus patients and the difficulty in clearly defining a meaningful and quantifiable clinical outcome are limiting the success of trials of promising and rationally designed biologics.

It is in the context of this new understanding of the central role of the IFN pathway, along with the urgent need for improved and biologically meaningful measures of disease characteristics and activity, that research to define lupus biomarkers is accelerating. In the previous issue of Arthritis Research \& Therapy, Fu and colleagues [1] presented crosssectional data that are based on a cohort of SLE patients, rheumatoid arthritis (RA) patients, and healthy donors and that support the potential utility of measuring chemokine transcripts in peripheral blood as an indicator of disease activity. Their study builds on a growing literature that has used microarray, real-time polymerase chain reaction, or functional assays of IFN- $\alpha$ activity to quantify activation of the IFN pathway [5-7]. The measurement of serum chemokine protein has been proposed as an additional approach to define biologically and clinically meaningful changes in disease activity $[8,9]$.

As chemokine gene transcription is 'downstream' of IFN- $\alpha$, the authors measured chemokine transcripts and related those data to various clinical parameters. A panel of seven chemokine genes was selected based on evidence of their induction by IFN- $\alpha$. It should be noted, however, that in contrast to the IFN-inducible genes that are highly specific for response to type I IFN, including those used in the study of $\mathrm{Fu}$ and colleagues to define an IFN score (such as IFIT1, IFIT3, MX1, OAS1, and Ly6e), the chemokine gene products studied (such as RANTES, MCP-1, MIG, IP-10, CXCL11, IL-8, and $C C L 19)$ can be induced by a wide variety of stimuli,

IFN = interferon; IL-8, interleukin-8; RA = rheumatoid arthritis; SLE, systemic lupus erythematosus. 
including IFN- $\gamma$, tumor necrosis factor, growth factors, and bacterial and viral products. The IFN score, then, is a fairly specific measure of type I IFN activity (or induction by a virus), while measurement of chemokine gene expression provides a biomarker that integrates the composite effects of multiple exogenous and endogenous stimuli, including type I IFN but also a range of other influences. For that reason, a panel of chemokine transcripts (or chemokine proteins) might be predicted to provide a measure of a broader complement of molecular influences that result in flares of disease activity or augmented inflammation in a target organ.

The data presented by $\mathrm{Fu}$ and colleagues [1] indicate that a composite score based on chemokine gene expression in peripheral blood leukocytes is significantly associated with lupus disease activity, as assessed by the SLEDAI-2K (Systemic Lupus Erythematosus Disease Activity Index 2000) or C3 levels. The chemokine score showed a slightly greater degree of correlation with disease activity than did an IFN score and was said to be more specific for SLE, as the IFN score was also elevated in RA patients. Among the promising data are those showing significantly higher chemokine scores in patients with active lupus nephritis compared with those with inactive lupus nephritis, although this effect was blunted in the patients treated with at least $30 \mathrm{mg}$ of prednisone daily. Nonetheless, the data suggest that the chemokine score might be a candidate for validation as a biomarker of those requiring aggressive immunosuppressive therapy for lupus nephritis.

While the IFN and chemokine gene expression scores were correlated with each other, the degree of correlation was weak, consistent with the chemokine score reflecting diverse stimuli, including but not restricted to IFN- $\alpha$. In addition, the presence of a chemokine score in some patients who did not express an IFN score implicates molecular pathways other than that induced by IFN in those patients. The data presented are consistent with previous studies that focused on serum chemokines and that also demonstrated an association with increased disease activity $[8,9]$.

Neither chemokine nor IFN-induced transcripts are likely to be useful as a diagnostic biomarker specific for SLE. Although relative specificity of increased chemokine transcript expression for SLE compared with RA was observed in the study of Fu and colleagues, many conditions, including infections and metabolic syndrome, also demonstrate increased expression of chemokines. IFN-inducible gene expression is increasingly recognized as a feature of a number of systemic autoimmune diseases, including systemic sclerosis, polymyositis, dermatomyositis, Sjögren syndrome, and RA. But measurement of IFN-inducible gene expression should be valuable for selection of patients and demonstration of effective blockade of IFN in clinical trials targeting that pathway and might predict future flares in some patients. In contrast, chemokine expression may be a useful measure of more generalized inflammation that suggests ongoing target organ damage.
Beyond their utility in defining relationships between immunologic mediators and clinical parameters, these biomarker data should stimulate further analysis of immunologic stimuli, receptors, and signaling pathways distinct from those that result in IFN pathway activation. For example, immune complexes derived from lupus patients with arthritis induce significantly higher levels of IL-8 than those from lupus patients without arthritis, a function that does not depend on the presence of DNA or RNA [10].

The demonstration of associations of chemokine and IFN scores with disease activity and, in some studies, lupus nephritis should stimulate multicenter longitudinal studies to determine the relative value of these scores, along with measurements of serum chemokine and IFN proteins or functional activity, as measures of disease activity over time. Validation of a biomarker useful for selection of patients for clinical trials, prediction of disease flares, or measurement of response to therapy could move lupus clinical research toward its holy grail: approval of effective new therapies.

\section{Competing interests}

Both authors have submitted a patent application for a type I interferon assay.

\section{References}

1. Fu Q, Chen X, Cui H, Guo Y, Chen J, Shen N, Bao C: Association of elevated transcript levels of interferon-inducible chemokines with disease activity and organ damage in systemic lupus erythematosus patients. Arthritis Res Ther 2008, 10:R112.

2. Medzhitov R, Preston-Hurlburt P, Janeway CA Jr.: A human homologue of the Drosophila Toll protein signals activation of adaptive immunity. Nature 1997, 388:394-397.

3. Lovgren T, Eloranta ML, Bave U, Alm GV, Ronnblom L: Induction of interferon-alpha production in plasmacytoid dendritic cells by immune complexes containing nucleic acid released by necrotic or late apoptotic cells and lupus IgG. Arthritis Rheum 2004, 50:1861-1872.

4. Crow MK: Type I interferon in systemic lupus erythematosus. Curr Top Microbiol Immunol 2007, 316:359-386.

5. Crow MK, Kirou KA, Wohlgemuth J: Microarray analysis of interferon-regulated genes in SLE. Autoimmunity 2003, 36:481490.

6. Kirou KA, Lee C, George S, Louca K, Peterson MG, Crow MK: Activation of the interferon-alpha pathway identifies a subgroup of systemic lupus erythematosus patients with distinct serologic features and active disease. Arthritis Rheum 2005, 52:1491-1503.

7. Hua J, Kirou K, Lee C, Crow MK: Functional assay of type I interferon in systemic lupus erythematosus plasma and association with anti-RNA binding protein autoantibodies. Arthritis Rheum 2006, 54:1906-1916.

8. Bauer JW, Baechler EC, Petri M, Batliwalla FM, Crawford D, Ortmann WA, Espe KJ, Li W, Patel DD, Gregersen PK, Behrens TW: Elevated serum levels of interferon-regulated chemokines are biomarkers for active human systemic lupus erythematosus. PLoS Med 2006, 3:e491.

9. Bauer JW, Petri M, Batliwalla FM, Wilson J, Slattery C, Singh S, Tesfasyone H, Gregersen PK, Behrens TW, Baechler EC: IFNregulated chemokines are reliable biomarkers of systemic lupus erythematosus. Arthritis Rheum 2007, 56:S782.

10. Hua J, Kirou KA, Crow MK: Distinct clinical manifestations in SLE are associated with interferon vs. inflammatory gene expression induced by distinct immune stimuli. Arthritis Rheum 2008, 58:S342. 